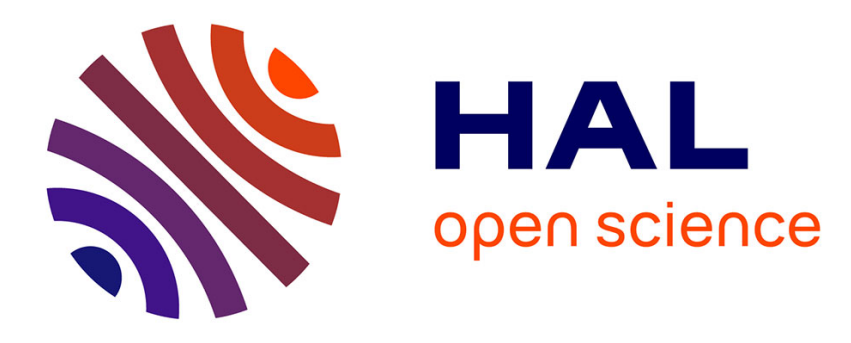

\title{
Réflexion éthique et institutionnalisation de l'éthique
}

Catherine Dekeuwer, R. Chvetzoff, C. Clouzeau, N. Kopp

\section{To cite this version:}

Catherine Dekeuwer, R. Chvetzoff, C. Clouzeau, N. Kopp. Réflexion éthique et institutionnalisation de l'éthique. Ethique \& santé, 2011, 8 (3), pp.125-131. 10.1016/j.etiqe.2010.10.004 . hal-02951258

\section{HAL Id: hal-02951258 \\ https://univ-lyon3.hal.science/hal-02951258}

Submitted on 12 Oct 2020

HAL is a multi-disciplinary open access archive for the deposit and dissemination of scientific research documents, whether they are published or not. The documents may come from teaching and research institutions in France or abroad, or from public or private research centers.
L'archive ouverte pluridisciplinaire HAL, est destinée au dépôt et à la diffusion de documents scientifiques de niveau recherche, publiés ou non, émanant des établissements d'enseignement et de recherche français ou étrangers, des laboratoires publics ou privés. 
Éthique et santé (2011) 8, 125-131 "Réflexion éthique et institutionnalisation de l'éthique » C. Dekeuwer ${ }^{a, *}$, R. Chvetzoffa,b ${ }^{\text {, C. Clouzeauc }}$, N. Kopp ${ }^{d}$

Résumé On assiste depuis 2002, en France, à une réorganisation du secteur de la santé pour une meilleure coordination et une qualité de la prise en charge des usagers. Dans ce contexte, on demande aux professionnels d'organiser, de mettre en œuvre et d'évaluer une démarche éthique. Celle-ci devient alors un objet d'évaluation requis par la procédure de certification de la HAS et les évaluations externes des établissements médicosociaux de I'ANESM. L'éthique devient obligatoire et on lui applique la logique de la démarche qualité. Cela est-il compatible avec les exigences d'une réflexion éthique? Comme la démarche qualité impose des impératifs de rationalisation pour une meilleure efficience de l'organisation, la démarche éthique ne risque-t-elle pas d'être réduite à une simple variable d'ajustement conduisant à la mise en œuvre de recommandations ou d'avis certifiés éthiques? Nous proposons ici de questionner cette démarche en identifiant deux dimensions de l'éthique rétives à toute institutionnalisation: l'exigence de pensée et l'éthique du soin. 


\section{Introduction}

Le secteur de la santé est en pleine mutation et réorganisation, comme en témoigne la création des Agences régionales de santé (ARS) [1]. Ces dernières ont notamment pour missions d'impulser un décloisonnement des secteurs sanitaire et médicosocial pour une prise en charge coordonnée, tant en amont qu'en aval du parcours de soins des patients, personnes âgées ou handicapées. Les enjeux sont à la hauteur de l'ampleur de cette réorganisation. Sont concernés près de 2800établissements d'hospitalisation pour le secteur sanitaire et quelques 38000 établissements ou structures médicosociales pour personnes âgées ou handicapées. Audelà des données quantitatives, les autorités de santé et plus particulièrement, la HAS et l'ANESM ${ }^{1}$ travaillent également sur l'amélioration de la prise en charge des usagers par une plus grande formalisation et harmonisation des pratiques des professionnels, un ancrage fort du droit des usagers (notamment pour une plus grande autonomie) [2-5], la prise en compte de la bientraitance, et enfin, la mise en place d'une réflexion éthique des professionnels de santé ${ }^{2}$ quant à leur pratique. C'est la difficulté de la mise en place de cette dernière que nous allons ici interroger et discuter.

Encadrés par la procédure de certification V2010de la HAS et les évaluations externes de l'ANESM ${ }^{3}$, les professionnels de santé doivent désormais organiser, mettre en œuvre et évaluer la réflexion éthique au sein de leur établissement. Or, si on ne peut que se réjouir de ce projet qui conduirait à plus de réflexion éthique, la question reste ouverte de savoir si la manière dont ces institutions la mettent en place et se proposent de l'évaluer n'est pas contraire à ce qu'on attend d'une réflexion éthique. On aboutit ainsi à une évaluation de la démarche éthique elle-même, et en l'occurrence de la manière dont les questions éthiques sont repérées et traitées. Mais est-ce que l'institutionnalisation de la réflexion éthique constitue une garantie du caractère éthique des décisions qui sont prises dans ces établissements? II ne sera pas question ici de nier les effets positifs de l'élaboration de recommandations de bonnes pratiques ou de la mise en place de démarches qualité au sein des établissements de soin [6]. Nous voulons insister sur les dimensions de la réflexion éthique rétives à une institutionnalisation commandée par les principes de la démarche qualité.

\section{Réalités de l’institutionnalisation de l'éthique}

La situation des professionnels de santé qui prennent en charge des personnes malades, âgées ou handicapées n'est pas facile. Ils sont, en effet, confrontés à un grand nombre de situations problématiques d'un point de vue éthique qui résultent, notamment, des progrès issus des techniques thérapeutiques. Leur situation est également marquée par un contexte juridique renforcé, notamment par la loi du 4mars2002relative aux droits des malades et à la qualité du système de santé [2] et celle du 2janvier2002rénovant l'action sociale et médicosociale [3]. Enfin, vivre dans une société où les valeurs

\footnotetext{
${ }^{1}$ La Haute Autorité de santé (HAS) a la charge du secteur sanitaire (hôpitaux, cliniques, soins de suite et de réadaptation, réseaux de santé) et l'Agence nationale de l'évaluation et de la qualité des établissements et services sociaux et médicosociaux (ANESM) le secteur médicosocial (handicap et gériatrie) et social (centres pour jeunes travailleurs, par exemple). La HAS et l'ANESM ont signé, le 14juin2010, un accord de collaboration afin de développer des actions communes ou complémentaires, notamment dans deux domaines: celui des recommandations de bonne pratique et celui de l'évaluation des établissements et services de santé, médicosociaux et sociaux. Cet article ne concerne pas le domaine social, mais les secteurs médicaux et médicosociaux.

${ }^{2}$ Seront dénommés ici et par souci de simplification professionnels de santé l'ensemble des professionnels issus du secteur sanitaire et médicosocial (professionnels médicaux, paramédicaux, éducateurs spécialisés, etc.).

${ }^{3}$ La procédure de certification de la HAS est une démarche obligatoire réalisée tous les quatre ans par les établissements sanitaires. L'évaluation externe concerne, quant à elle, les établissements et services sociaux ou médicosociaux. Deux évaluations externes doivent être réalisées par des Cabinets habilités par l'ANESM entre la date de l'autorisation et son renouvellement.
} 
ne vont plus de soi, où le relativisme et le pluralisme sont généralement de mise, conduit nombre de professionnels de santé à une demande de réflexion éthique.

L'ANESM, dans sa lettre de mission Recommandation de bonnes pratiques professionnelles: l'éthique dans les établissements et services sociaux et médicosociaux, explique: la réflexion éthique émerge avec une acuité particulière au sein d'un conflit de valeurs d'égale légitimité.

Par exemple, une question éthique se pose lorsque, à l'occasion d'une situation concrète, le droit à la liberté se heurte à l'impératif de sécurité; ou encore, lorsque la nécessité du partage de l'information rentre directement en conflit avec le devoir de confidentialité; une autre configuration classique est celle de l'écart possible entre le respect de la volonté de la personne et l'impératif d'intervention [7]. On reconnaît ici des situations concrètes: la liberté d'aller et de venir de personnes âgées qui peut être dangereuse pour elles-mêmes et autrui, la question de savoir comment entendre et respecter un refus de soin aux conséquences vitales pourtant parfois dramatiques, la difficulté dans certains cas de respecter le secret médical et la confidentialité - dont l'importance est rappelée par la loi du 4 mars2002- lorsque la vie d'autrui est potentiellement en danger.

Plus qu'un conflit entre deux types de valeurs, il s'agit aujourd'hui de poser les questions éthiques de deux manières différentes, qu'on peut appeler l'éthique de l'autonomie et l'éthique du soin ${ }^{4}$. II est en effet question, dans cette citation, de droits et de valeurs qui prennent sens dans le contexte d'une éthique déontologique qui place l'autonomie des personnes au premier plan de l'action et de la réflexion. Mais il est fait référence à un autre régime éthique, dont le principe est la réponse aux besoins de la personne vulnérable: mettre en place les conditions de sa sécurité ou intervenir pour l'alimenter, par exemple. L'articleL116-1du code de l'action sociale et des familles créé par la loi 2002-2du 2janvier2002 [3] fait également référence, dans ses principes généraux, à ces deux manières de poser les questions éthiques: L'action sociale et médicosociale tend à promouvoir, dans un cadre interministériel, l'autonomie et la protection des personnes (...). Elle repose sur une évaluation continue des besoins et des attentes des membres de tous les groupes sociaux, en particulier des personnes handicapées et des personnes âgées, des personnes et des familles vulnérables, en situation de précarité ou de pauvreté.

Dans la mesure où les fondements théoriques et les conséquences pratiques de ces éthiques ne sont souvent pas distingués (alors qu'ils peuvent aboutir à des conséquences contradictoires), il ne serait pas surprenant que ce manque de clarté aboutisse à des injonctions contradictoires pour les soignants.

Une réponse institutionnelle est aujourd'hui apportée aux difficultés que peuvent rencontrer, dans ce contexte, les professionnels de santé: la mise en œuvre d'une réflexion éthique devient pour eux une obligation, avec les démarches de certification des établissements sanitaires et celles de l'évaluation externe des établissements sociaux et médicosociaux. En effet, la HAS, qui a notamment la charge de certifier l'ensemble des établissements sanitaires, impose dans le manuel de certification (critère 1c) [13] que les hôpitaux intègrent dans leur projet d'établissement la dimension éthique de la prise en charge.

\footnotetext{
${ }^{4}$ Ces deux éthiques sont souvent opposées [8, introduction et 9]. Nous avons préféré ne pas identifier l'éthique du soin aux éthiques du care. Certes, les mots de besoin, vulnérabilité et protection renvoient aux questions auxquelles les éthiques du care ont apporté une réponse. Mais dans la mesure où le courant du care n'est pas unifié et que son identification à une éthique du soin est problématique [10,11], nous avons choisi de laisser la plus ouverte possible, pour l'explorer, la référence au soin. Dans la dernière partie de cet article, nous envisagerons, en revanche, une conception du care défendue par Tronto et Paperman [12].
} 
De même, ils doivent s'assurer que l'ensemble des professionnels soit sensibilisé et formé à l'éthique et que les questions éthiques soient repérées et traitées. L'ANESM travaille, quant à elle, à l'élaboration d'une recommandation de bonnes pratiques professionnelles intitulée L'éthique dans les établissements et services sociaux et médicosociaux [7]. La non-prise en compte et la non-formalisation de la réflexion éthique par les professionnels peuvent désormais avoir des répercussions fortes sur l'établissement, tant pour son image que pour sa réputation. En effet, pour le secteur sanitaire, les rapports de certification sont mis en ligne sur le site de la HAS et sont donc accessibles par tous, professionnels et usagers. Pour le secteur médicosocial, il est fort probable que les rapports d'évaluation externe soient bientôt accessibles sur le site de l'ANESM.

La manière dont l'éthique est actuellement institutionnalisée repose sur la mise en place d'une évaluation de la réflexion éthique par le biais de la démarche qualité. Nous voyons alors apparaître une évaluation non pas des pratiques mais de la démarche éthique, soumise aux principes de l'efficacité et de la reproductibilité. En effet, la méthodologie de mise en place des démarches d'amélioration continue de la qualité est appliquée à l'éthique: on organise la réflexion éthique au sein de l'établissement, on la met en place, on l'évalue et on ajuste les pratiques en fonction des résultats (telle la roue de Deming de la démarche qualité: Plan, Do, Check, Act). Par exemple, le critère 1c du Manuel de certification de la HAS [13] conduit à une évaluation de la démarche éthique dans le cadre de la procédure de certification. II faut en effet prévoir dans le projet d'établissement de prendre en compte la dimension éthique de la prise en charge (étape1). II faut ensuite mettre en œuvre le traitement des questions éthiques (étape2). Enfin, il faut évaluer et améliorer la démarche éthique de l'établissement en faisant l'état des lieux des questions éthiques (étape3). Or, par définition, une démarche qualité repose sur des impératifs de rationalisation et vise à l'efficacité, et à l'efficience (le meilleur niveau de qualité au moindre coût). La reproductibilité des événements et des démarches est également une condition de possibilité de l'évaluation. Il s'agit donc désormais de s'assurer que les questions éthiques sont traitées efficacement et cette efficacité doit être évaluée par les professionnels de l'établissement, d'une part, et les experts visiteurs de la HAS, d'autre part.

\section{Difficultés de l'institutionnalisation de l'éthique}

L'expérience de terrain met en évidence qu'il existe une réelle difficulté pour les professionnels dans le repérage et le traitement des questionnements éthiques, que l'institutionnalisation de l'éthique ne fait malheureusement que renforcer.

Pour une infirmière de Maison d'accueil spécialisée, la problématique éthique est au cœur même de la prise en charge des personnes handicapées du fait même de vouloir bien faire; l'éthique relève plus pour cette infirmière d'une approche individuelle que collective et n'engage pas de processus théorique. Une infirmière d'un hôpital de soins de longue durée pour personnes âgées affirme, concernant la prise en charge des patients en fin de vie, qu'elle fait systématiquement appel au réseau ou à l'équipe mobile de soins palliatifs pour résoudre les problématiques éthiques; la réflexion éthique de l'infirmière est déléguée à d'autres professionnels de santé considérés comme les spécialistes de la fin de vie. 
Un éducateur spécialisé d'un Foyer d'accueil médicalisé considère son travail comme éthique car il peut prouver que les outils de la loi du 2 janvier2002 sont élaborés et correctement mis en œuvre (livret d'accueil, projet personnalisé, contrat de séjour, charte des droits de la personne handicapée, etc.). Un cadre de santé d'un hôpital général de type médecine chirurgie obstétrique présente, non sans une certaine fierté, les formulaires et procédures relatifs aux directives anticipées, à la personne de confiance pour les patients en fin de vie [5]. Dans les deux cas, le respect de la loi civile tend à se substituer à la réflexion morale. Certains juristes expliquent que les lois sont faites précisément pour répondre à des problèmes éthiques ou promouvoir certaines valeurs, comme la dignité des personnes. Rien d'étonnant donc à ce que suivre un protocole défini par la loi soit conforme à l'éthique et heureusement pourrait-on dire.Ainsi, les difficultés d'application de certaines lois sont parfois l'occasion de réflexions éthiques qui donnent sens aux pratiques. Mais ici, l'éthique est au contraire réduite à une application sans réflexion de la loi.

Selon un directeur d'hôpital, faire sortir rapidement les patients de son établissement et donc réduire la fameuse durée moyenne de séjour (DMS), relève d'une approche éthique car cela permet à d'autres patients d'être pris en charge par l'établissement; pour ce directeur, l'approche collective des soins pour le plus grand nombre de patients l'emporte sur les considérations individuelles là où la réflexion éthique chercherait au moins à concilier intérêts individuels et collectif. Enfin, un médecin de réseau de soins palliatifs aura scrupuleusement élaboré avec son équipe une check-list éthique qui reprend rigoureusement l'ensemble des aspects réglementaires et organisationnels nécessaires à une prise en charge éthique et de qualité; l'éthique est pensée et mise en œuvre selon une approche procédurale.

Ces exemples nous amènent à distinguer cinq effets des procédures actuelles qui vont à l'encontre de la réflexion éthique:

- l'éthique relève plus de la pratique habituelle que de la réflexion;

- l'éthique est réduite à l'obéissance à la loi civile;

- l'éthique est réduite à l'application d'une procédure;

- l'éthique est confiée à des experts ou des professionnels de l'éthique;

- à force d'être confrontée à des problèmes collectifs, l'éthique est réduite à un utilitarisme dévoyé.

En même temps, force est de constater que certaines postures décrites ici renvoient à un aspect essentiel de la dimension éthique des pratiques de soin. Le soin, par exemple, est souvent pensé comme une disposition à l'action qui prend en compte les besoins de l'autre plutôt que comme une application de principes éthiques ${ }^{5}$. Patricia Paperman explique ainsi que penser l'éthique comme s'il s'agissait d'un élément extérieur aux pratiques, une dimension surajoutée (comme une couche de vernis), c'est faire une erreur d'un type particulier: une erreur de catégorie [12, p.90]. Autrement dit, les manières dont les réponses aux besoins sont dispensées et organisées constituent une dimension éthique des pratiques. Par ailleurs, la procédure en elle-même, la considération de la collectivité et le cadre des lois civiles ne

\footnotetext{
${ }^{5}$ La disposition n'est pas contraire à la réflexion mais se distingue du jugement déterminant consistant à appliquer des principes généraux à des situations particulières.
} 
sont pas contraires à la réflexion l'éthique; le défaut à souligner est celui du réductionnisme, c'està-dire la réduction de la réflexion éthique à l'application de procédures, de règles ou de lois. L'habitude, la volonté de bien faire et le fait que la loi conduise notre regard dans certaines directions sont donc autant d'éléments qui rendent difficile le retour critique sur les pratiques. Quant à la résolution des problèmes, force est de constater que la loi fournit des solutions qui masquent parfois leur difficulté d'application et tendent à remplacer la réflexion par une procédure. Finalement, l'institutionnalisation de la réflexion éthique met en avant, par sa méthodologie qui relève de la démarche qualité, le respect de procédures réitérables, l'obligation d'être conforme à une norme générale et la gestion efficace d'un collectif. Elle ne fait donc que renforcer ces cinq effets contraires à la réflexion éthique.

Ces exemples invitent à considérer enfin deux séries de difficultés. Il existe peut-être une tendance à la généralisation du souci de l'éthique dans le secteur de la santé à laquelle semble répondre l'institutionnalisation de l'éthique. Mais le mot éthique, alors survalorisé, semble apporter une valeur ajoutée à l'action alors qu'on peut se demander ce qu'il signifie. N'est-ce pas l'action d'institutionnaliser l'éthique qui est valorisée plutôt que les réflexions des professionnels? On peut aller jusqu'à penser que l'éthique finit par absorber et unifier des normes de comportements qui relèveraient plus de simples manières de se comporter humainement, par exemple, en respectant les droits des usagers ou en écoutant la plainte d'un patient.Elle va donc à l'encontre de ce que nous appelons l'exigence de pensée.

Deuxièmement, face à la stratégie délibérée de mise en œuvre de standards éthiques, les professionnels de santé adoptent une nouvelle stratégie de type organisationnelle, administrative et juridique, qui a tendance à se substituer à la réflexion éthique, individuelle et collective, mise en œuvre dans une situation singulière. Alors même que la réflexion éthique impose de réfléchir en prenant en compte la singularité de la situation, l'universalité des exigences éthiques et la particularité des lois civiles, elle devient application d'une procédure certifiée éthique. La réflexion éthique, dont le nom n'est plus finalement qu'un simple label, se réduit alors à l'application de démarches dérivées de la logique démarche qualité. En ce sens, elle paraît irréconciliable avec l'éthique des soins, dont le principe est l'attention à la vulnérabilité toujours unique. La difficulté est donc posée: tout en reconnaissant le caractère essentiel d'une réponse à la vulnérabilité, la HAS et l'ANESM ne s'engagent pas vers la réponse la plus adaptée.

Nous aimerions donc présenter l'exigence de pensée et l'éthique du soin comme deux orientations fondamentales de l'éthique et en interroger le caractère institutionnalisable.

\section{L'exigence de pensée ne peut être institutionnalisée}

Un des principaux écueils de l'institutionnalisation de l'éthique est de la réduire à une simple procédure d'application des lois civiles ou de bonnes mœurs. Kant, puis Arendt, ont souligné cet écueil. Revenir sur leurs arguments nous permet de mieux comprendre pourquoi l'exigence de pensée, fondamentale pour la réflexion, ne peut être institutionnalisée. Précisons que cette proposition ne signifie pas que les institutions n'ont pas à encourager la réflexion éthique; elle signifie que l'institutionnalisation de la réflexion éthique ne peut pas garantir le caractère éthique ou non des décisions qui sont prises. 
Institutionnalisée, la réflexion éthique peut n'être qu'une coquille vide et même conduire à l'aveuglement les professionnels de santé.

Selon Kant ${ }^{6}$, suivre une règle de conduite selon la lettre mais non selon l'esprit peut conduire au moralisme: se tenir pour justifié moralement ou considérer sa pratique comme éthique pourvu qu'une règle de conduite n'ait pas été clairement transgressée. Ces règles de conduites peuvent être définies par la loi civile ou par la moralité commune (les bonnes mœurs). Or, ce moralisme est dangereux parce qu'il conduit à étouffer en l'homme la réflexion proprement morale. Après tout, si nos actions sont conformes à ce que prescrivent les règles de conduite habituelles, légales et morales, pourquoi vouloir aller plus loin dans l'interrogation sur le sens de nos actes et la nature de nos intentions? Autrement dit, il est d'autant plus facile de ne pas s'interroger sur nos véritables intentions que des règles sont prêtes à être appliquées.

L'institution de recommandations de bonnes pratiques en éthique et la recherche d'une certification éthique conduisent à instaurer ce qui constitue, aujourd'hui et dans le domaine du soin, de bonnes mœurs.

Demain, un soignant pourra appliquer ce qui deviendra, selon la démarche qualité, un protocole éthique. Par chance, il pourra ne jamais rencontrer de situations où l'esprit s'oppose à la lettre. Il pourra se sentir moralement justifié, par définition pourrait-on dire, puisqu'il suit un protocole certifié éthique. Mais cette situation ne le conduira-t-elle pas encore plus sûrement qu'aujourd'hui à une absence de questionnement sur ses intentions morales et sur le sens de ses pratiques? L'écueil serait de produire une bonne conscience fondée sur l'application de protocoles certifiés éthiques, là où le cheminement plus difficile du questionnement engageant individuellement sur les intentions et le sens des pratiques serait de mise. Cela ne signifie pas que les réflexions communes, la dimension collégiale et collective des décisions n'a pas de sens ou de valeur. Cela veut dire que la réflexion éthique ne peut pas se passer de sa dimension proprement individuelle, d'un engagement et d'une responsabilité vis-à-vis de soi-même et de ses propres intentions morales.

Aujourd'hui, la réflexion éthique pourrait être disqualifiée ou considérée comme inutile en raison de l'existence de démarches certifiées éthiques. À bien réfléchir, tout le monde conviendrait sans doute qu'au lieu d'endormir les consciences morales par l'application de procédures, il conviendrait de les éveiller à la pensée. Mais Arendt explique pourquoi cet éveil ne saurait être institutionnalisé. Pour elle, la pensée est la condition de possibilité des jugements sur le bien et le mal, mais elle ne saurait être institutionnalisée et ce pour deux raisons.

D'abord, les conditions dans lesquelles l'homme pense, selon Arendt, ne correspondent pas aux conditions requises aujourd'hui par les autorités de santé pour que les soignants puissent résoudre des problèmes pratiques. Selon Arendt, l'exercice de la pensée est en lui-même solitaire ${ }^{7}$, il nécessite d'être dégagé des contraintes matérielles et pratiques, il n'a pas pour fin l'action et enfin, il ne donne naissance à aucune règle, serait-elle provisoire. Cela ne signifie pas que les comités d'éthique ou les recommandations de bonne pratique ne soient pas utiles ni conformes aux règles morales. Mais cela

\footnotetext{
${ }^{6} \mathrm{Cf}$. La religion dans les limites de la simple raison, première dissertation [14].

${ }^{7}$ Cela ne signifie pas que les échanges et les réflexions collectives ne nourrissent pas notre pensée.
} 
indique que la condition de possibilité des jugements sur le bien et le mal, c'est-à-dire la pensée, relève d'un autre type d'activité intellectuelle, qui ne saurait être oublié.

Deuxièmement, la pensée est dangereuse: la pensée a inévitablement un effet minant, destructeur, sur tous les critères établis, les valeurs et mesures du bien et du mal; en bref, sur ces coutumes et règles de conduite dont on traite en morale et en éthique [15, p.51]. Les règles morales, les lois civiles et les recommandations de bonne pratique nous permettent de vivre en société et d'agir. Mais appliquer ces règles à la lettre peut être dangereux. Au niveau individuel, cette attitude peut conduire à la mise en sommeil de la conscience morale ${ }^{8}$. Au niveau collectif, Hottois souligne dans cette perspective un risque de récupération des comités de bioéthique en comités de morale instruments d'un pouvoir théocratique ou idéologique et totalitaire [17, p.42]. La pensée ne peut pas être institutionnalisée: l'exigence de pensée revient à la conscience personnelle, qui seule peut être en rapport avec elle-même et donner sens à ses engagements. Libre dialogue de l'âme avec ellemême, elle ne produit rien d'autre qu'ellemême et ne vaut que comme activité toujours recommencée. Nos institutions démocratiques ont, quant à elles, la tâche de ne pas l'étouffer. En allant jusqu'au bout de cette perspective, on peut donc penser que l'institutionnalisation de la réflexion éthique est un contre-sens qui peut s'avérer dangereux si les professionnels de santé en viennent à estimer que toute l'éthique se réduit à l'application d'un ensemble de procédures prédéfinies. D’où viendra alors le vent de la pensée $[15$, p.52] nécessaire à l'engagement éthique personnel et à la démocratie?

\section{Quel type d'institutionnalisation pour une éthique du soin?}

Notre seconde critique porte plus spécifiquement sur l'institutionnalisation en tant qu'elle prend la forme de la démarche qualité, supposant donc uniformisation, généralisation, reproductibilité, recherche d'efficacité et d'efficience, mais également de sécurité et de gestion des risques. Ce type de démarche nous paraît contraire à ce que requiert une éthique du soin, alors même que l'institutionnalisation de l'éthique est actuellement mise en œuvre dans les lieux de prise en charge de la plus grande vulnérabilité. La loi du 4mars2002et la HAS concernent essentiellement des personnes malades. La loi du 2janvier2002et l'ANESM concernent l'aide sociale à l'enfance, la prise en charge du handicap, de la vieillesse, des enfants délinquants, de jeunes qui présentent des difficultés d'adaptation, etc. C'est donc une éthique du soin, en tant qu'elle répond à la vulnérabilité, qui devrait être mise en œuvre. La difficulté est alors de savoir comment l'éthique du soin pourrait être institutionnalisée. À vrai dire, l'argument selon lequel l'éthique du soin ne peut pas être institutionnalisée car le care vise à répondre à chaque fois à la demande et aux besoins singuliers d'un individu n'est pas suffisant. La thèse que nous souhaitons défendre ici est plus nuancée: il s'agit de montrer que c'est en adoptant le point de vue et les exigences de ce type d'éthique que la question de son institutionnalisation pourrait être posée. Mais elle serait alors posée hors de la démarche qualité. Pour première définition d'une éthique du soin, nous choisissons pour guide la contribution de Paperman [12] au volume collectif Qu'est-ce que le care? Souci des autres, sensibilité, responsabilité. Elle a le mérite, d'abord, de rappeler que l'éthique du care ne doit pas être confondue avec un supplément d'âme qui

\footnotetext{
${ }^{8}$ Dans les Considérations morales [15], Arendt prend l'exemple de Eichmann, dont elle a suivi le procès en 1961 (Eichmann à Jérusalem. Rapport sur la banalité du mal [16]). Eichmann était chargé de régler tous les problèmes concernant le transport des juifs vers les camps de la mort. Elle note qu'Eichmann, tout en appliquant un système de valeurs et en utilisant les catégories du bien et du mal, se caractérise par son absence de pensée.
} 
accompagnerait les gestes techniques relatifs au soin (habiller, nourrir, poser un cathéter, prescrire un médicalement, par exemple). Elle parvient, de plus, à dégager le care des pratiques privées pour en révéler la dimension publique, ce qui nous permet de mieux discuter de son institutionnalisation. L'éthique du care ne vise pas à proposer, comme on l'imagine parfois, la pitié et la compassion, la sollicitude ou la bienveillance comme des vertus subsidiaires adoucissant une conception froide des relations sociales, ou ajoutant un supplément d'âme à une conception impartiale de la justice basée sur la primauté des droits d'individus autonomes séparés et rationnels [12, p.94]. Les réflexions sur le care en font un concept plus précis et plus exigeant que la reconnaissance de la nécessité d'une dimension plus humaine des rapports soignant-soigné. Les relations de soin sont, en effet, comprises dans leur dimension sociale, et en particulier, le care est considéré comme un travail.

Comment son institutionnalisation dans le cadre d'établissement de santé peut-il avoir un sens? Démontrons d'abord que cela ne peut être dans le contexte d'une démarche qualité. Le care demande, en effet, une attention soutenue aux autres: il répond à des besoins qui émergent à chaque fois de situations singulières, complexes et non généralisables. Il est donc impossible d'appliquer la démarche qualité à l'éthique du care parce qu'elle est relative à des situations qui ne sont pas reproductibles. Le care est également considéré comme difficile à identifier et à conceptualiser, ce qui rend impossible son évaluation dans le cadre d'une démarche qualité. Enfin, il repose sur un sens moral qui se rapporte à la qualité des relations et à l'attention aux besoins d'un autre individu. Le point d'achoppement principal nous paraît être le suivant: le care mobilise d'autres capacités morales que l'apprentissage de principes ou de règles éthiques. Les spécialistes parlent de sens moral et expliquent que dans certaines situations, la sensibilité morale peut s'aiguiser progressivement. Le bon soin s'apprendrait par expérience, dans la discrétion et le silence de la pratique.

Comme l'explique Marie Garrau: Ia professionnalisation pouvait porter atteinte à la qualité du care mis en œuvre. C'est le cas quand elle va de pair avec la standardisation des pratiques de care, voire avec leur soumission progressive à des normes d'efficacité et de productivité qui heurtent de front la dimension éthique et l'engagement affectif que les travailleurs de care placent au centre de leur travail et qui sont difficilement quantifiables [9]. La critique est double: d'une part, par définition, le care ne peut être standardisé. D'autre part, de cette institutionnalisation du care peut découler une plus grande vulnérabilité des soignants et non seulement des patients. Cette dernière critique fait écho aux plaintes que l'on peut entendre du côté des praticiens, dont l'investissement est à la fois exigé et en même temps, non reconnu par l'institution.

Toutes ces difficultés ne signifient pas pour autant que l'éthique du care doive rester du domaine du privé, ni qu'il n'y ait pas nécessité à le penser le care comme un travail, avec la dimension d'un questionnement sur la justice sociale que cela comporte. II convient au contraire de réfléchir sur les conditions sociales et institutionnelles ainsi que sur la politique qui permettraient de garantir les conditions les plus justes pour ce travail spécifique et, en conséquence, la meilleure qualité de relation possible. Si l'on souhaite donner au soin une place dans le domaine public et l'institutionnaliser, il convient de reconnaître ses spécificités et de le reconnaître comme une forme de travail à part entière, avec ses propres contraintes et spécificités. Or, la question de la plus juste et la plus appropriée distribution du travail du soin devrait se poser comme une question éthique relative au care, c'est-à-dire au souci des relations et au souci de la vulnérabilité des soignés et des soignants. 
La question quelles doivent être nos relations de sorte que leur nature et leur qualité soient telles qu'elles puissent répondre à nos vulnérabilités? est encore une question qui manifeste le souci des relations. Elle ne peut recevoir de réponse dans une démarche qualité.

\section{Conclusion}

L'institutionnalisation de l'éthique, telle qu'elle est mise en œuvre actuellement par la démarche qualité, peut-elle répondre à la demande de réflexion éthique exprimée par les professionnels de la santé et la société? Adaptée aux spécificités des secteurs sanitaire et médicosocial, l'éthique pourrait certes être mise en œuvre au sein d'un établissement ou d'un service à partir d'un questionnement sur le sens originel de ses missions. Mais le souci éthique ne se résume pas à la bonne exécution des pratiques professionnelles quotidiennes, à l'obéissance à la loi civile, à une délégation de pensée à d'autres professionnels choisis es qualité, à des procédures ou des protocoles, ou à des arguments faussement utilitaristes. Au contraire, le risque de moralisme associé à l'adoption d'une démarche de certification éthique est important. Si rien ne doit inciter les professionnels à renoncer au devoir de se maintenir dans une position constante de réflexion, on peut craindre cependant que l'existence de démarches certifiées éthiques conduise à l'endormissement des consciences. L'éthique est une réflexion inlassable sur le sens de ce que l'on fait et sur le rapport à l'autre. Si trop peu d'éthique conduit à l'immoralité et à l'inhumanité, trop d'éthique, au sens actuel, risque de conduire inversement à la bonne conscience qui rend aveugle aux vraies exigences de la moralité. Par ailleurs, la nécessité de faire une place à une éthique du soin est confusément énoncée par les autorités de santé. Nous avons montré la nécessité d'organiser une réflexion sur la manière dont nous voulons, collectivement, laisser place aux pratiques de soin et institutionnaliser l'éthique du soin. Cependant, il apparaît que la démarche qualité ne saurait être à la hauteur de cette tâche.

\section{Conflit d'intérêt}

Aucun conflit d'intérêt.

\section{Références}

[1] Loi n² 2009-879 du 21 juillet 2009 portant réforme de l'hôpital et relative aux patients, à la santé et aux territoires, http://www.legifrance.gouv.fr/.

[2] Loi ${ }^{\circ}$ 2002-303 du 4 mars 2002 relative aux droits des malades et à la qualité du système de santé, http://www.legifrance.gouv.fr/.

[3] Loi $\mathrm{n}^{\circ}$ 2002-2du 2 janvier 2002 rénovant l'action sociale et médicosociale, http://www.legifrance.gouv.fr/.

[4] Loi n² 2005-102 du 11 février 2005 pour l'égalité des droits et des chances, la participation et la citoyenneté des personnes handicapées, http://www.legifrance.gouv.fr/.

[5] Loi no 2005-370 du 22 avril 2005 relative aux droits des malades et à la fin de vie http://www.legifrance.gouv.fr/.

[6] Freudiger S, Pittet G, Christen-Gueissaz E. Convergences et décalages entre la portée institutionnelle des démarches qualités et le bien-être des résidents d'établissements médicosociaux certifiés. Éthique en santé 2007;4:4-11. 
[7] ANESM. Lettre de cadrage. Recommandation de bonnes pratiques professionnelles L'éthique dans les établissements et services sociaux et médicosociaux. www.ANESM.sante.gouv.fr; 2009.

[8] Molinier P, Laugier S, Paperman P. Qu'est ce que le care? In: Souci des autres, sensibilité, responsabilité. Paris: Petite bibliothèque Payot; 2009, 298p.

[9] Garrau M. Éthiques et politiques du care. In: Bourdeau V, Merrill R (Eds.). DicoPo, Dictionnaire de théorie politique; www.dicopo.org.

[10] Benaroyo L, Lefève C, Mino JC, et al. La philosophie du soin. In: Éthique, médecine, société. Paris: PUF; 2010, 352p.

[11] Worms F. Le moment du soin. Paris: PUF; 2010, 250p.

[12] Paperman P. D'une voie discordante: désentimentaliser le care, démoraliser l'éthique. In: Molinier P, Laugier S, Paperman P, editors. Qu'est-ce que le care? Souci des autres, sensibilité, responsabilité. Paris: Petite bibliothèque Payot; 2009. p. 89-110.

[13] Direction de l'amélioration de la qualité et de la sécurité des soins. Manuel de certification des établissements de santé V2010. Paris: Éd. Haute Autorité de santé; 2009, 98p.

[14] Kant E. La religion dans les limites de la simple raison. CEuvres philosophiques. T3. Paris: Gallimard coll. Pléiade; 1986, 1561p.

[15] Arendt H. Considérations morales. Paris: Rivages poche; 1996, 77p.

[16] Arendt H. Eichmann à Jérusalem. Rapport sur la banalité du mal. Paris: Gallimard Folio; 1997, 484p.

[17] Hottois G. L'éthique en comités. J Int Bioethique, 2007;18: 35-46. 